\title{
Noise in Brain Activity Engenders Perception and Influences Discrimination Sensitivity
}

\author{
Fosco Bernasconi, ${ }^{1,2 \star}$ Marzia De Lucia, ${ }^{3,4 \star}$ Athina Tzovara, ${ }^{3,4 \star}$ Aurelie L. Manuel, ${ }^{1,2}$ Micah M. Murray, ${ }^{1,3,4,5}$ \\ and Lucas Spierer ${ }^{1,6}$ \\ ${ }^{1}$ Neuropsychology and Neurorehabilitation Service, Department of Clinical Neurosciences, ${ }^{2}$ Department of Psychiatry, Community Psychiatry Service, and \\ ${ }^{3}$ Radiology Department, Vaudois University Hospital Center and University of Lausanne, 1011 Lausanne, Switzerland, ${ }^{4}$ Electroencephalography Brain \\ Mapping Core, Center for Biomedical Imaging, 1011 Lausanne, Switzerland, ${ }^{5}$ Department of Hearing and Speech Sciences, Vanderbilt University Medical \\ Center, Nashville, Tennessee 37232-0014, and ${ }^{\circ}$ Neurology Unit, Medicine Department, University of Fribourg, 1700 Fribourg, Switzerland
}

Behavioral and brain responses to identical stimuli can vary with experimental and task parameters, including the context of stimulus presentation or attention. More surprisingly, computational models suggest that noise-related random fluctuations in brain responses to stimuli would alone be sufficient to engender perceptual differences between physically identical stimuli. In two experiments combining psychophysics and EEG in healthy humans, we investigated brain mechanisms whereby identical stimuli are (erroneously) perceived as different (higher vs lower in pitch or longer vs shorter in duration) in the absence of any change in the experimental context. Even though, as expected, participants' percepts to identical stimuli varied randomly, a classification algorithm based on a mixture of Gaussians model (GMM) showed that there was sufficient information in single-trial EEG to reliably predict participants' judgments of the stimulus dimension. By contrasting electrical neuroimaging analyses of auditory evoked potentials (AEPs) to the identical stimuli as a function of participants' percepts, we identified the precise timing and neural correlates (strength vs topographic modulations) as well as intracranial sources of these erroneous perceptions. In both experiments, AEP differences first occurred $\sim 100$ ms after stimulus onset and were the result of topographic modulations following from changes in the configuration of active brain networks. Source estimations localized the origin of variations in perceived pitch of identical stimuli within right temporal and left frontal areas and of variations in perceived duration within right temporoparietal areas. We discuss our results in terms of providing neurophysiologic evidence for the contribution of random fluctuations in brain activity to conscious perception.

\section{Introduction}

Behavioral and brain responses to identical stimuli can vary depending on experimental parameters, for example, which physical dimension of the stimulus observers are instructed to attend (Rinne et al., 2009) or the context of stimulus presentation (van Dijk and Backes, 2003). In the absence of any experimental manipulation, spontaneous variations in attention also dramatically impact behavioral and brain responses to ambiguous stimuli (Haynes et al., 2005; Sterzer et al., 2009). However, in all these cases, percepts are based on and reflect an actual physical characteristic of the stimuli. For instance, in binocular rivalry paradigms, different images are presented to each eye and attention

\footnotetext{
Received July 20, 2011; revised Sept. 26, 2011; accepted 0ct. 22, 2011.

Author contributions: F.B. and L.S. designed research; F.B. and A.L.M. performed research; F.B., M.D.L., A.T., and L.S. analyzed data; F.B., M.M.M., and L.S. wrote the paper.

This research was funded by grants from the Pierre Mercier Foundation for Science (to L.S.) as well as by the Swiss National Science Foundation (grants K-33K1_122518/1 to M.D.L. and 310030B-133136 to M.M.M.). Cartool software (http://sites.google.com/site/fbmlab/cartool) has been programmed by Denis Brunet, from the Functional Brain Mapping Laboratory, Geneva, Switzerland, and supported by the Center for Biomedical Imaging of Geneva and Lausanne. We thank Ayse At for her help in collecting of the data.

${ }^{*}$ F.B., M.D.L., and A.T. contributed equally to this work.

Correspondence should be addressed to Lucas Spierer, Faculty of Biology and Medicine, University of Lausanne, Neuropsychology and Neurorehabilitation Service, Vaudois University Hospital Center, av. Pierre-Decker 5, 1011 Lausanne, Switzerland. E-mail: lucas.spierer@gmail.com.

DOI:10.1523/JNEUROSCI.3715-11.2011

Copyright $\odot 2011$ the authors $\quad 0270-6474 / 11 / 3117971-11 \$ 15.00 / 0$
}

modulates which of the two images is consciously perceived (Tong et al., 2006).

Here, we hypothesize that differential brain responses to identical sensory stimulation also occur in the absence of any variation in the experimental context or in attention, due to random fluctuations in brain activity ("brain noise"; Faisal et al., 2008; Neri, 2010) that may exceed additional sources of external noise. Computational models suggest that due to noise, the trial-to-trial variations in brain responses to identical stimuli could be large enough to themselves cause perceptual differences (Micheyl et al., 2009; Neri, 2010). Signal detection theory (SDT) posits that the magnitude of noise along sensory processing pathways corresponds to the ratio between an observer's sensitivity to differences along a given stimulus dimension and the minimal physical distance between two different stimuli required to perceive them as different at an above-chance level (i.e., the discrimination threshold; Macmillan and Creelman, 2005). SDT also inherently assumes negligible contributions from external sources of noise. In the case of auditory frequency discrimination, SDT predicts a substantial probability for two physically identical stimuli to elicit brain responses that are sufficiently different to induce a perception of pitch difference (Micheyl et al., 2009). That is, identical stimuli could often be perceived as differing in pitch based on the "false" activation of neuronal populations due to the noise combining/interacting with the response to the physical stimulation. 
Numerous evidence documents that noise arises at levels ranging from the transduction of physical to nervous signal (Lillywhite and Laughlin, 1979) to neural response (de Ruyter van Steveninck et al., 1997; Faisal et al., 2008).

To address whether trial-to-trial fluctuations in brain responses yield measurable differences between responses to identical stimuli perceived as different, we recorded high-density EEG during a two-alternative forced-choice discrimination paradigm on pairs of identical auditory stimuli. While being kept naive that the two stimuli of each pair were actually identical, participants were instructed to report which of the two stimuli was higher in pitch [Experiment (Expt.) 1] or longer in duration (Expt. 2). To identify the contributions of random variations in brain activity to conscious perception and discrimination abilities, we first tested whether it was possible to predict subjective perception at a single-trial level based on topographic information. Second, we performed electrical neuroimaging analyses of auditory evoked potentials (AEPs) to the identical sounds averaged as a function of the subjective percepts to identify the spatiotemporal networks underlying subjective discrimination.

\section{Materials and Methods Participants}

Sixteen right-handed participants (eight females; mean age \pm SEM: $27.6 \pm 1.1$ years) participated in Experiment 1. Ten right-handed participants (five females; $24.5 \pm 1.1$ years) participated in Experiment 2. None had a history of neurological or psychiatric illness. All reported normal hearing. Handedness was assessed with the Edinburgh questionnaire (Oldfield, 1971). Each participant provided written, informed consent to procedures approved by the local ethics committee and in accordance with the Declaration of Helsinki.

\section{Task design}

We conducted two experiments, each involving a psychophysics discrimination task on physically different stimuli (Expts. 1a and 2a) and an EEG discrimination task on identical stimuli (Expts. $1 \mathrm{~b}$ and 2b). Expt. 1 involved pitch discrimination, and Expt. 2 involved duration discrimination. Expts. 1a and 2a served to ensure that participants were aware of what pitch and duration differences referred to and to measure participants' pitch- and duration-discrimination thresholds. Expts. $1 \mathrm{~b}$ and $2 \mathrm{~b}$ served to investigate the neural correlates of the processing of identical stimuli presented during a discrimination task with the instruction of discriminating between the pitch (Expt. 1) or duration (Expt. 2) of the identical stimuli.

In Expts. $1 \mathrm{~b}$ and 2b, pairs of 150-ms-duration, $756 \mathrm{~Hz}$ tones (sinusoids, $10 \mathrm{~ms}$ rise/fall time; $44.1 \mathrm{kHz}$ digitization; generated using Adobe Audition 2.0) were presented separated by a constant stimulus onset asynchrony of $800 \mathrm{~ms}$. In Expt. 1a, the sounds were $750 \mathrm{~Hz}$ and $756 \mathrm{~Hz}$ tones with duration of $150 \mathrm{~ms}$. In Expt. 2a, the sounds were $756 \mathrm{~Hz}$ tones of 150 and $129 \mathrm{~ms}$ duration. The sounds were presented via insert earphones (model ER-4P; Etymotic Research).

In Expts. 1 and 2, the tasks were two-alternative forced-choice discrimination paradigms in which participants were presented with pairs of sounds and instructed to indicate (using the index and middle fingers of their right hand) if the first or second sound was of a higher frequency (Expt. 1) or of longer duration (Expt. 2). No time limit was given to the participants for responding. Participants were naive to the aims of the study and were not aware that stimuli were actually identical in Expts. $1 \mathrm{~b}$ and 2b. In Expt. 1a, participants completed one block of 30 sound pairs (trials). In Expt. 1b, participants completed 10 blocks of 108 trials. In Expt. 2a, participants completed one block of 32 trials. In Expt. 2b, participants completed three blocks of 80 trials.

During the tasks, participants fixated a white central cross on a black background. In Expts. 1b and 2b, a visual feedback, consisting of a randomly chosen green (correct response) or red (incorrect) square (500 ms duration), was presented $300 \mathrm{~ms}$ after the response. To avoid that partic- ipants suspected that the discrimination was actually impossible, we presented a positive feedback for two-thirds of the trials in half of the 10 blocks and in one-third of the trials in the remaining five blocks. The next trial was presented 700-1000 ms after the end of the feedback. In Expt. 1a and $2 \mathrm{a}$, no feedback was presented. Stimulus delivery and participants' responses were controlled by Eprime 2.0 software (Psychology Software Tools).

\section{Behavioral analyses}

Behavioral data of Expts. 1a and 2a, in which participants had to discriminate between stimuli physically differing in pitch or duration, respectively, were analyzed according to signal detection theory (Green and Swets, 1966). Sensitivity $\left(d^{\prime}\right)$ was calculated according to Macmillan and Creelman (2005), using the formula $d^{\prime}=z$ (Hits) $-z$ (FA), where FA is false alarms. Hits were High-Low and Long-Short (HL/LS) sound pairs reported as High-Low and Long-Short, and false alarms were Low-High and Short-Long (LH/SL) pairs reported as High-Low and Long-Short, respectively. Because the same number of HL/LS and LH/SL pairs were presented, the $d^{\prime}$ is symmetric: Hits + Misses $=$ False Alarms + Correct Rejections $=100 \%$. Therefore, the $d^{\prime}$ would be identical if accurately perceived LH/SL pairs were considered as Hits and HL/LS trials reported $\mathrm{LH} / \mathrm{SL}$ as False Alarms. Consequently, the $d^{\prime}$ index takes into account the global behavioral performance, encompassing accuracy to both HL/LS and LH/SL pairs. Accuracy cannot be assessed for Expts. $1 \mathrm{~b}$ and $2 \mathrm{~b}$ because the sounds in each pair were identical and thus there was no correct response. The $C$ criterion $(C=-0.5[z($ Hits $)+z(\mathrm{FA})])$ was calculated to assess whether there was any response bias during the psychophysical experiments.

Behavioral data of Expts. $1 \mathrm{~b}$ and $2 \mathrm{~b}$, in which participants had to discriminate between identical stimuli, were analyzed to determine whether there was any bias in response pattern. Because the discrimination tasks were actually impossible, participants could have adopted response strategies and consequently, their behavior and the resulting analyses of ERP would have been driven by high-order decision-making processes instead of being determined by random variations (i.e., brain noise) as we postulate. To address this possibility, we conducted a response pattern analysis by calculating the autocorrelation function of the two types of responses (High/Low Pitch and Long/Short Duration). This analysis enabled us to determine whether responses were actually random or whether there was any kind of pattern in the response scheme of the participants. If response patterns are indeed driven by random fluctuations, there should be no autocorrelation. To compare the sequences of the subject with randomly generated sequences, we generated random sequences of digits " 1 " and " 2 " of the same length as those of the subjects' responses for each block and each experiment. For the random sequences and for each subject, we computed the correlation of the response type in each trial $n$ with that in trial $n+m$ for $m=1-30$ (for a similar approach, see Britz et al., 2009, 2011).

\section{EEG acquisition and preprocessing}

Continuous EEG was acquired at $1024 \mathrm{~Hz}$ through a 128-channel Biosemi ActiveTwo AD-box referenced to the common mode sense (CMS; active electrode) and grounded to the driven right leg (DRL; passive electrode), which functions as a feedback loop driving the average potential across the electrode montage to the amplifier zero. In Expt. 2, because 8 of the 10 participants were recorded with 64 channels, EEG data of the two participants recorded with 128 channels were downsampled to the common 64 channels across all participants before data processing in Expt. 2. For the single-trial analysis, EEG data from both experiments were downsampled to 64 channels to reduce the computational load.

Data preprocessing and analyses were performed using Cartool (Brunet et al., 2011). Peristimulus epochs of EEG (spanning - $100 \mathrm{~ms}$ to 500 $\mathrm{ms}$ after stimulus onset) were averaged from each participant to calculate ERPs for the higher/longer and lower/shorter perceived pitch/duration conditions. A semiautomated $\pm 80 \mu \mathrm{V}$ artifact rejection criterion was applied. For Expt. 1b, the average number of accepted epochs from a given individual participant for the high (mean \pm SEM; $936.9 \pm 16.8$ ) and low $(932.9 \pm 18.5)$ perceived pitch conditions were not significantly 
different $(p>0.5)$. For Expt. 2b, the average number of accepted epochs from a given individual participant for the long (mean \pm SEM; $224 \pm 7$ ) and short $(224.3 \pm 7)$ perceived duration conditions were not significantly different $(p>0.8)$. The number of trials that were the first or second sound within the pair of stimuli presented on a given trial and designated to each perceptual condition did not differ significantly (Expt. 1b: $p>0.1$; Expt. $2 \mathrm{~b}: p>0.2$ ), ensuring that our effects did not follow from mere differences in priming or habituation effects. This result likewise indicates that participants did not exhibit any bias in reporting which of the two identical sounds was perceived as higher in pitch (Expt. 1b) or longer in duration (Expt. 2b). Before the single-trial analysis and group averaging, data from artifact electrodes of each participant were interpolated (Perrin et al., 1987), bandpass filtered $(0.18-40.0 \mathrm{~Hz})$, and recalculated against the average reference.

\section{EEG analyses}

General analysis strategy

To determine the contribution of random fluctuations in brain activity to conscious perception, we analyzed AEPs recorded during the discrimination tasks between identical stimuli both at the single-trial and at average AEP levels.

We first examined whether specific patterns of brain activity in response to the identical sounds predicted the way participants perceived each sound relative to the other sound of the pair (either of higher of lower pitch in Expt. $1 \mathrm{~b}$ or of longer or shorter duration in Expt. 2b). This was done principally for the following reasons. In the presence of identical stimuli, it is not possible to quantify behaviorally whether participants perceived identical sounds on a given trial. As detailed in the Results section, there were in fact equal numbers of trials where the first stimulus of the pair was perceived as higher versus lower in pitch (Expt. 1b) or longer versus shorter in duration (Expt. 2b), indicating that participants' subjective percepts of each trial (or at least their reporting) was overall random. Therefore, one way to evaluate participants' subjective reports is to predict their response at the single-trial EEG level. By predicting participants' percepts from single-trial EEG, we ensured that the measured variations in brain responses to the identical stimuli were actually linked to perception, thereby establishing that brain noise modulates conscious perception. In other words, if the percepts can be predicted at above-chance levels from the scalp-recorded electrophysiological responses, it indicates that the fluctuations in the brain responses to the identical sounds are indeed related to the variations in the subjective percepts of the stimuli's dimensions attended to by the participants. We based our single-trial classification scheme on voltage topographies (De Lucia et al., 2007, 2010; Murray et al., 2009; Tzovara et al., 2011a) because of their neurophysiological interpretability: a change in electric field topography necessarily follows from a change in the configuration of the underlying generators (Lehmann, 1987). The classification was conducted for each participant separately to obtain a distinct (although possibly overlapping) model of topographies for each participant.

After determining whether the percepts could be classified based on the EEG responses, we sorted the EEG responses as a function of the percepts and computed an average AEP for each percept condition. By increasing the signal-to-noise ratio, the computation of AEPs enabled us to reveal where and when in the brain random activity was related to variations in perceived pitch or duration. Moreover, while single-trial classification is performed for each subject separately, AEPs analysis allows to evaluate the underlying mechanism of subjective perception at the group level. Mean AEPs evoked by sounds that were judged to be higher in pitch were compared to mean AEPs evoked by sounds that were judged to be lower in pitch as detailed in the "AEP analyses" section below. The same approach was applied in Expt. $2 \mathrm{~b}$ where the same stimuli and paradigm were used, but participants were instead instructed to discriminate between the perceived sound duration. Specifically, mean AEPs from sounds that were judged to be longer in duration were compared to mean AEPs from sounds that were judged to be shorter in duration regardless of whether the sound was presented first or second within a trial (the same number of first and second sounds of a pair were included in the ERP of each percept condition).

\section{Single-trial classification analyses}

We first extracted a set of voltage topographies representative of the whole dataset (template maps). This was achieved by computing mixtures of Gaussians models (GMMs) using only one part of all the available trials (training dataset). Full details of this procedure are explained and validated in Tzovara et al. (2011a). The GMM allows us to cluster groups of similar topographies together and therefore to reduce the original dataset to a small number of few representative topographies or "template maps." This clustering was performed for each experimental condition separately (i.e., sounds perceived as lower or higher pitch in Expt. $1 \mathrm{~b}$ and as shorter or longer duration in Expt. $2 \mathrm{~b}$ ), providing us with one set of template maps per condition (i.e., the mean of each cluster).

The GMMs were then used for classifying test trials based on posterior probabilities. In other words, we computed the posterior probability that the topographies of a test trial are represented by the template maps within the GMMs for the two conditions. To maximize the discrimination between the two conditions in the test dataset, the posterior probabilities were only computed over temporal periods for which the two conditions differed significantly across trials in the training dataset and only for the template maps that were responsible for this difference. A test trial was then assigned to the condition (e.g., lower or higher pitch in Expt. 1b) whose template maps yielded the highest posterior probability. The accuracy of classification was evaluated by measuring the area underlying the receiver operating characteristic curve (AUC) (Macmillan and Creelman, 2005). A perfect classification yields an AUC value of 1 , while a complete misclassification gives an AUC of 0 .

To limit the computational time, we considered single trials extracted from 2 of the 10 blocks of Expt. 1b (the fourth and eighth), resulting in total number of trials ranging from 167 to 209 trials per condition and subject. For Expt. 2b, we considered all the trials from the three blocks (their total number ranged between 163 and 229 per condition and subject). We used 120 trials per condition for computing the GMMs through a 10-split cross-validation procedure. In each split, the training dataset comprised $90 \%$ of all the available trials and the test the remaining $10 \%$, in a way such that the test datasets across splits did not overlap. Overall, we computed 10 models, one for each split of the data, and we kept the one that yielded the highest AUC value across the 10 splits.

We finally estimated the predictive power of the single-trial model on a validation dataset, including the remaining trials for each participant (63 trials on average for Expt. $1 \mathrm{~b}$ and 113 for Expt. 2b). These trials were not used at any point in the cross-validation procedure and provide an objective measure of our algorithm's performance on completely new data. To assess chance levels, we randomly relabeled the validation trials with perceptual outcomes (e.g., lower or higher pitch in Expt. 1b), and the classification accuracy was reestimated 100 times. In this way, we obtained a distribution of values representing chance levels, and the AUC found in the validation dataset was tested against them $(t$ test; $p<0.01)$.

\section{AEP analyses}

Voltage waveform analyses. A first level of analysis was performed by comparing, using pairwise $t$ tests, AEPs to the high versus low perceived pitch (Expt. 1) and to the long versus short perceived duration (Expt. 2) conditions at each scalp electrode as a function of peristimulus time. The results of this analysis are displayed as an intensity plot where the $x$-axis represents time, the $y$-axis the scalp electrodes, and the intensity ( $z$-axis) the results of the $t$ tests. Note that only effects meeting or exceeding the $p<0.05$ criterion for at least 11 consecutive data points $(10 \mathrm{~ms})$ were considered reliable (Guthrie and Buchwald, 1991). We included this analysis here to give a visual impression of specific effects within the dataset and to facilitate the contextualization of our results with other AEP studies. However, due to the electrode reference-dependent nature of statistical analyses of AEP waveforms (statistical outcomes will thus change with the choice of the reference electrode; Tzovara et al., 2011b), our primary analyses and basis for interpretation were the electrode reference-independent analyses detailed below.

Global electric field analyses. Two reference electrode-independent analyses of the AEPs were conducted. Changes in the strength of the electric field were quantified using the global field power (GFP) (Murray et al., 2008; Koenig and Melie-García, 2010) from each subject and ex- 
perimental condition. The GFP is calculated as the standard deviation of the potentials at all electrodes and at each instant in time. This measure indicates the global strength of the response, regardless of its topographic distribution. Changes in GFP were statistically analyzed at each time point using a paired $t$ test between the high and low perceived pitch (Expt. 1b) and between the long and short perceived duration (Expt. 2b) conditions.

Topographic modulations were identified using global dissimilarity (DISS) (Lehmann and Skrandies, 1980), which is calculated as the root mean square of the difference between two strength-normalized vectors (here the instantaneous voltage potentials across the electrode montage). The DISS value between the high and low perceived pitch (Expt. 1b) and between the long and short perceived duration (Expt. 2b) conditions was then compared at each time point with an empirical distribution derived from a bootstrapping procedure (5000 permutations per data point) based on randomly reassigning each participant's data to either the high or low perceived pitch (Expt. 1b) or long or short perceived duration (Expt. 2b) condition (detailed in Murray et al., 2008). DISS is independent of the reference electrode and is insensitive to pure amplitude modulations across conditions (i.e., DISS modulations are orthogonal to GFP modulations). The utility of this analysis in terms of neurophysiologic interpretability is that topographic changes necessarily follow from differences in the configuration of the brain's underlying active generators (Lehmann, 1987). As above, temporal autocorrelation was corrected through the application of a $>11$ contiguous data-point temporal criterion for the persistence of differential significant effects (Guthrie and Buchwald, 1991). The results of the GFP and DISS analyses are displayed as $1-p$ value ( $y$-axis) as a function of time ( $x$-axis), with periods of significant differences highlighted in green.

Source estimations. We estimated the electrical activity in the brain using a distributed linear inverse solution applying the local autoregressive average (LAURA) regularization approach, comprising biophysical laws as constraints (Grave de Peralta Menendez et al., 2001, 2004; Michel et al., 2004). For the lead field matrix calculation, we applied the spherical model with anatomical constraints (SMAC) method (Spinelli et al., 2000), which transforms the MRI to the best-fitting sphere using homogeneous transformation operators. It then determines a regular grid of 3005 solution points in the gray matter of this spherical MRI and computes the lead field matrix using the known analytical solution for a spherical head model with three shells of different conductivities as defined by Ary et al. (1981).The results of the above topographic analysis provide an estimation of the time intervals when one can carry out source estimations. Statistical analyses of source estimations were performed by first averaging the AEP data across the period of interest to generate a single data point for each participant and condition. The inverse solution ( 16 participants $\times 2$ conditions for Expt. $1 \mathrm{~b}$ and 10 participants $\times 2$ conditions for Expt. 2b) was then estimated. Paired $t$ tests were calculated at each solution point using the variance across participants. Only nodes with $p$ values $<0.03$ two-tailed and clusters of at least 21 contiguous nodes were considered significant. This spatial criterion was determined using the AlphaSim program (http://afni.nimh.nih. gov/afni/doc/manual/AlphaSim). The results of source estimations were rendered on the Montreal Neurologic Institute's average brain with the Talairach and Tournoux (1988) coordinates.

\section{Results}

\section{Behavior}

In Expt. 1a (pitch-discrimination task between $750 \mathrm{~Hz}$ and 756 Hz tones), mean sensitivity $\left(d^{\prime}\right)=1.73 \pm 0.3$ (mean \pm SEM). In Expt. 2a (duration-discrimination task between $150 \mathrm{~ms}$ and 129 ms tones), $d^{\prime}=1.64 \pm 0.15$. These results indicate that participants were able to perceive subtle, though veridical, pitch and duration differences and knew to which sound characteristic to attend during the subsequent EEG discrimination task on physically identical stimuli.

For Expt. 1b involving pitch discrimination between identical tones, the first sound was reported as being of higher pitch on
$50.98 \pm 2.15 \%$ of trials. For Expt. $2 \mathrm{~b}$ involving duration discrimination between identical tones, the first sound was reported as being of longer duration on $47.9 \pm 2.45 \%$ of trials. These results provide no evidence for a systematic response bias or confounding effect of sound order. In addition, they suggest that the brain activity modulating the pitch or duration perception impacted randomly each of the two sounds of the pairs. Importantly, after Experiments $1 \mathrm{~b}$ and $2 \mathrm{~b}$, all participants reported being convinced that the two identical stimuli differed in either pitch or duration, respectively. This provides phenomenological support for the contention that brain responses to the identical tones included sufficient random activity to engender different percepts along the pitch and duration dimensions. However, although participants were actually convinced to have perceived differences in pitch and in duration at each trial, their overall response pattern was random (first sound reported as being "higher"/"longer" $50 \%$ of the trials). We therefore tested whether it was possible to predict at single-trial level subjective perception (see Single-trial analysis section). The $C$ criteria $(C=-0.5[z$ (Hits $)+z(\mathrm{FA})])$ for the pitch and duration psychophysical experiments were $0.27 \pm$ 0.11 (mean \pm SEM) and $-0.16 \pm 0.07$, respectively, providing no evidence of response bias.

For both Expts. $1 \mathrm{~b}$ and $2 \mathrm{~b}$, the autocorrelation curves were not distinguishable from what was obtained based on a random series (for a similar approach, see Britz et al., 2009, 2011), indicating that there was no patterning in the sequence of behavioral responses, as a cognitive bias would predict. Rather, the response patterns were completely random, for each task and each participant, speaking in favor of noise being at the origin of participants' responses (Fig. 1a,b).

\section{Single-trial analysis}

In Experiment 1b, the single-trial topographic algorithm predicted participants' responses with an accuracy in the validation dataset ranging between 0.48 and 0.59 (Fig. $2 a$, red dots). These accuracy values were above chance levels in 10 of the 16 participants ( $t$ test; $p<0.01$ ) (Fig. $2 a$, unmasked values). Average accuracy across these 10 participants was $0.56 \pm 0.01$ (mean \pm SEM) in the validation dataset and $0.57 \pm 0.01$ in the test dataset (Fig. $2 a$, blue dots).

In Experiment 2b, the single-trial topographic algorithm predicted participants' responses in the validation dataset with an accuracy that ranged between 0.47 and 0.62 (Fig. $2 b$, red dots). These accuracy values were above chance levels in 5 of the 10 participants ( $t$ test; $p<0.01$ ) (Fig. $2 b$, unmasked values). Average accuracy across these five participants was $0.55 \pm 0.01$ in the validation dataset and $0.60 \pm 0.01$ in the test dataset (Fig. $2 b$, blue dots).

This finding indicates that for the majority of the participants ( 15 of 26 , considering the two experiments together), there was sufficient information in scalp topography to predict above chance levels whether the identical stimuli were perceived as being higher in pitch (Expt. 1) or longer in duration (Expt. 2).

\section{AEP waveform analysis}

For Expt. 1b, mean AEPs evoked by sounds that were judged higher in pitch were compared to mean AEPs evoked by sounds that were judged lower in pitch. For Expt. 2b, mean AEPs evoked by sounds that were judged to be of longer duration were compared to mean AEPs evoked by sounds that were judged to be shorter in duration. Timewise statistical analyses comparing the two conditions in Expts. $1 \mathrm{~b}$ and $2 \mathrm{~b}$ were performed as a function of peristimulus time at each of the scalp electrodes (Figs. $3 a, 4 a$ ). 


\section{a Analysis of the sequence of response choice in the Pitch Experiment}

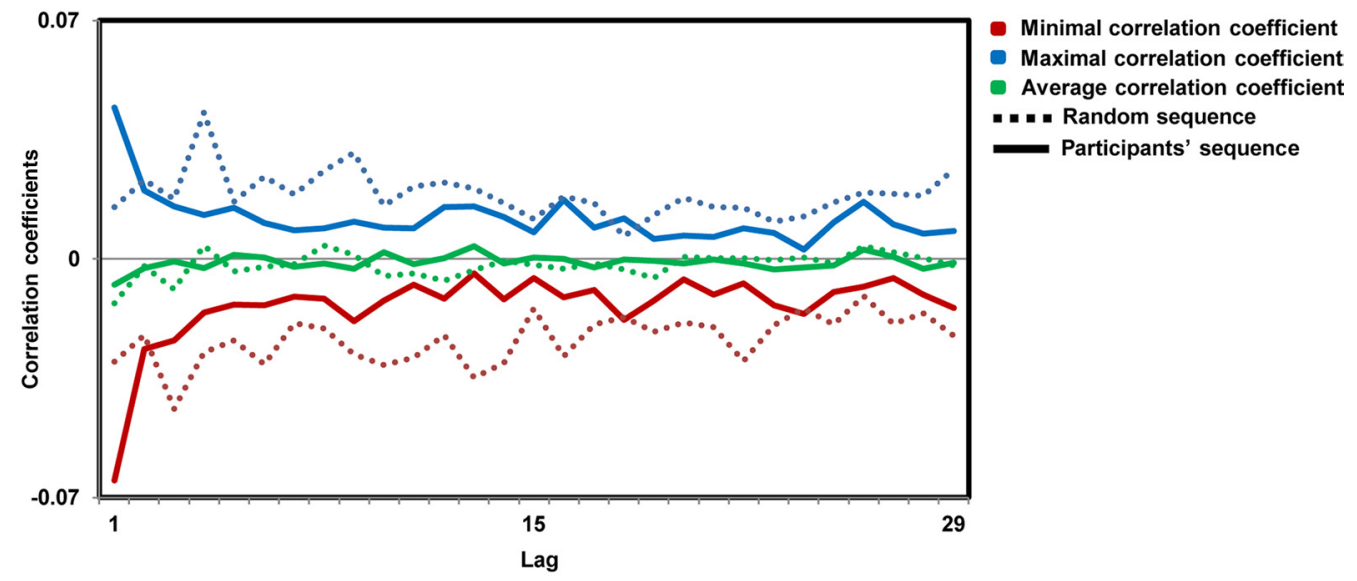

b Analysis of the sequence of response choice in the Duration Experiment

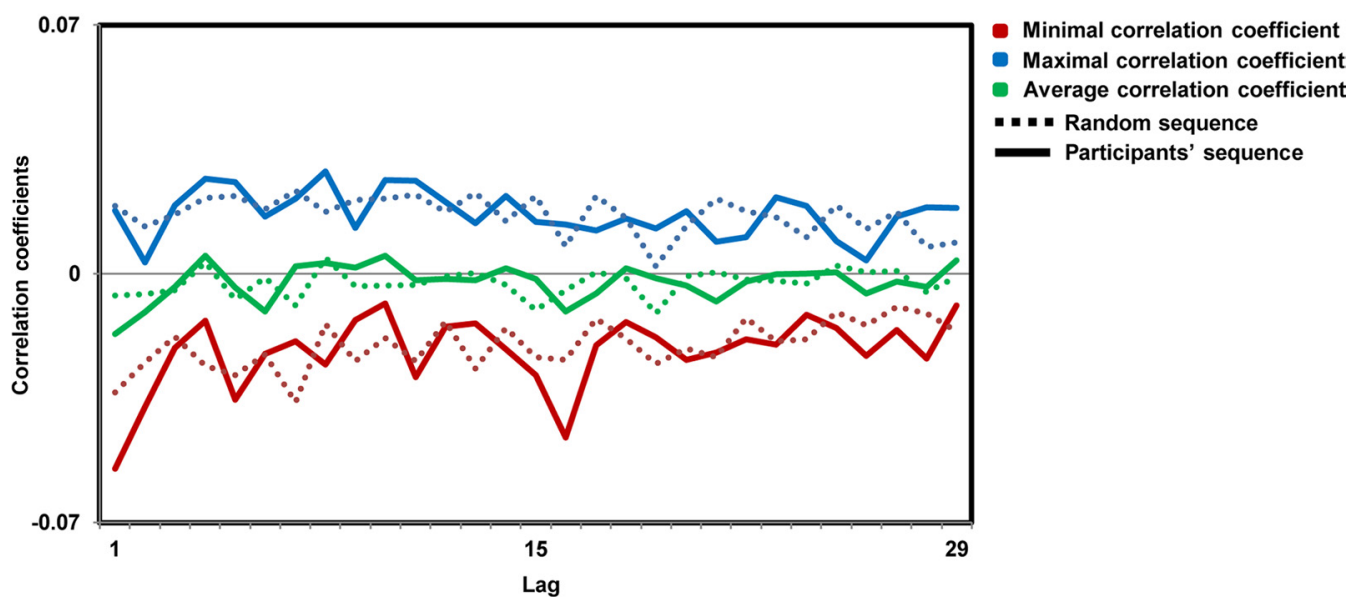

Figure 1. Analysis of the sequence of response choice. Autocorrelation coefficients are represented for the lags 1-30 for the pitch (a) and the duration (b) experiments. Maximal (blue lines), minimal (red lines), and average (green lines) correlation coefficients across participants are depicted for the participants' data (solid line) and for a random sequence (dotted line).

Statistically significant differences $(p<0.05,>10 \mathrm{~ms}$ ) were observed beginning $\sim 90-120 \mathrm{~ms}$ after stimulus onset over right frontal and left parietal electrodes for Expt. 1b and 100-140 ms after stimulus onset over left frontotemporal electrodes for Expt. $2 \mathrm{~b}$. We would remind the reader at this stage, however, that our conclusions regarding the likely causes of variations in perceived pitch or duration are based solely on analyses of electrode reference-independent features of the global electric field at the scalp.

\section{Global electric field analyses}

Reference-independent analyses differentiated modulations in electric field response strength (global field power, which would be consistent with a modulation in response gain or quantitative change in brain responses) versus topographic changes (global dissimilarity, which would be consistent with a modulation in the underlying brain sources or qualitative change). The GFP was contrasted at each time point between the high and low perceived pitch conditions (Expt. 1b; Fig. 3b) and between the long and short perceived duration conditions (Expt. 2b; Fig. 4b) using paired $t$ tests. There was no evidence for changes in response strength as a function of perceived pitch over time and modulations at $320 \mathrm{~ms}$ and $390 \mathrm{~ms}$ after stimulus as a function of perceived duration. By contrast, the nonparametric DISS statistics revealed significant topographic modulations over the 93-109 ms poststimulus interval for Expt. 1b (Fig. 3c) and 103-141 ms poststimulus interval for Expt. 2b (Fig. 4c), indicative of distinct configurations of active brain networks as a function of the perceived pitch or duration of the acoustically identical sounds. The results of the dissimilarity analysis served as the basis for the selection of the time period submitted to source estimations for Expt. $1 \mathrm{~b}$ and 2b, separately.

\section{Source estimations}

LAURA distributed source estimations were calculated over the period showing significant topographic differences between high and low perceived pitch or between long and short perceived duration, as revealed by the global dissimilarity analysis. To do this, AEPs for each participant and each experimental condition separately were averaged across the above-mentioned time period when topographic modulations were identified. Source estimations were then calculated and statistically compared using pairwise $t$ tests at the single-node level within the lead field matrix distributed throughout the gray matter of the MNI brain.

For Expt. 1b, sources underlying the above topographic modulations were estimated over the 93-109 ms poststimulus period using the LAURA inverse solution. There was a significantly stronger activation $\left(p<0.03 ; k_{\mathrm{E}}=21\right.$ solution points) within the left middle temporal and right middle frontal areas for stimuli perceived as lower versus higher pitch (Fig. 3d). 


\section{a Single-Trial Perceived Pitch Prediction for Sixteen Participants}

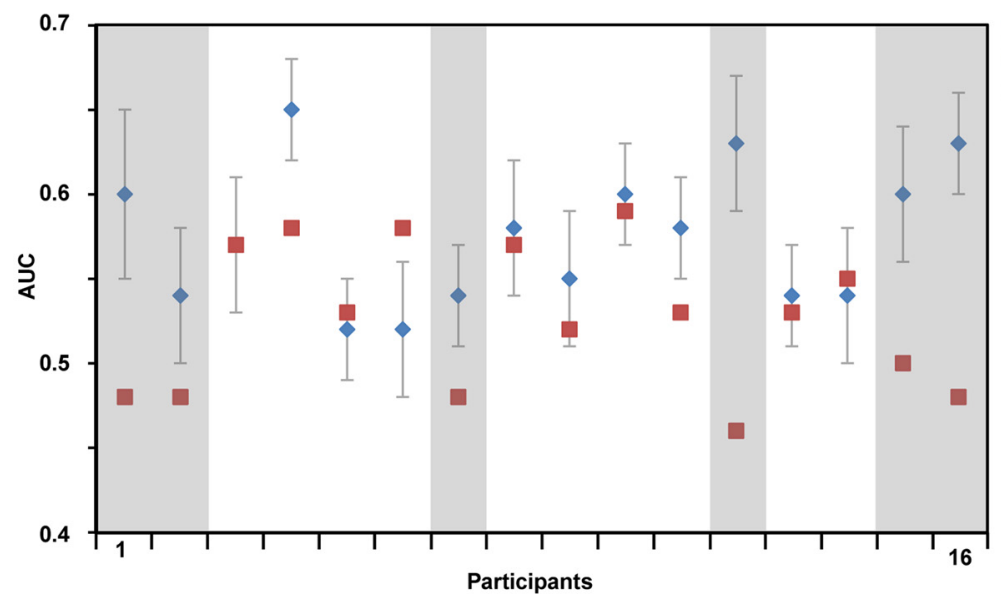

- Average AUC value in Validation dataset

- AUC value in Test dataset

- Non-significant prediction

\section{b Single-Trial Perceived Duration Prediction for Ten Participants}

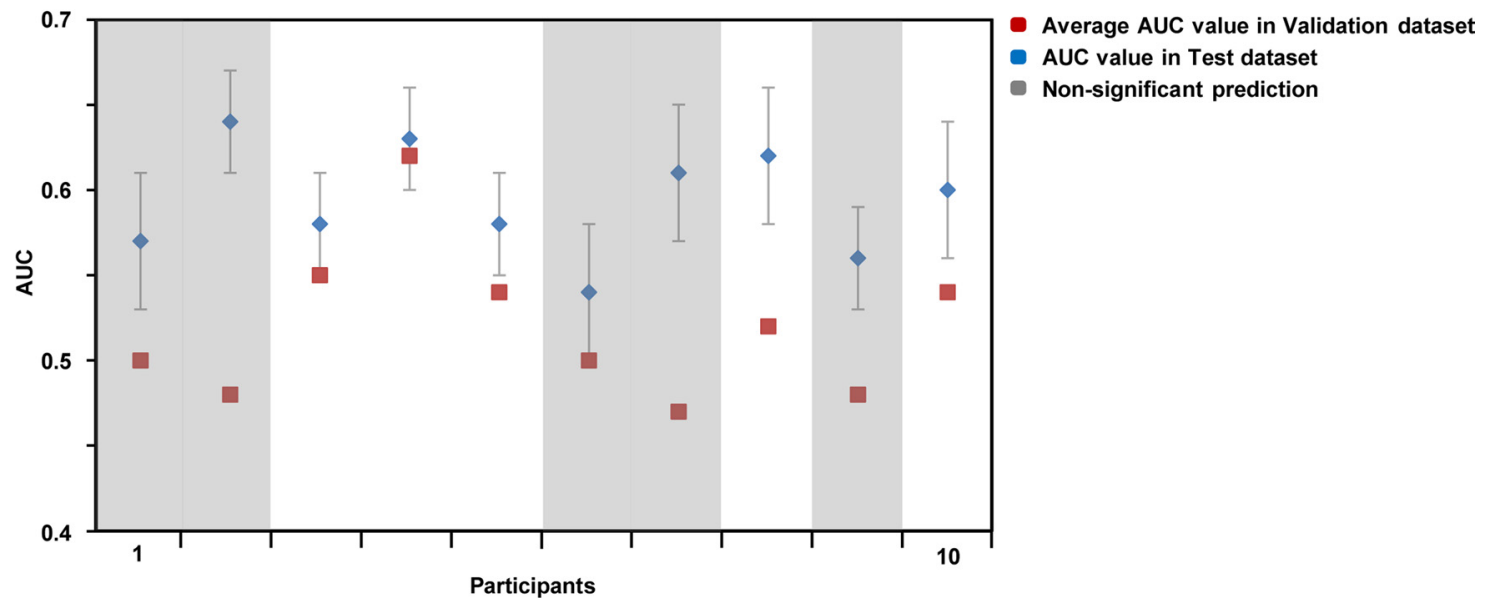

Figure 2. Single-trial EEG classification results for the pitch-discrimination experiment (Expt. 2a) and for the duration-discrimination experiment (Expt. 2b). Blue dots, Average values of the AUC across 10 splits of the data for each of the 16 (pitch experiment) or 10 participants (duration; \pm SEM across the 10 splits); red dots, AUC values on a validation dataset for each participant. The validation AUC values provide an objective measure of our algorithm's performance on completely new trials. Masked values (gray) indicate AUC values on the validation dataset that did not differ from chance level (participants 1, 2, 7, 12, 15, 16 for the pitch and participants 1, 2, 6, 7, 9 for the duration experiments).

For Expt. 2b, sources underlying the above topographic modulations were estimated over the 103-141 ms poststimulus period using the LAURA inverse solution. There was a significantly stronger activation $\left(p<0.03 ; k_{\mathrm{E}}=21\right.$ solution points) within right temporoparietal areas for stimuli perceived as shorter versus longer duration (Fig. 4d).

We then conducted correlation analyses between the mean of the scalar values of the solution points showing the differential activity across the experimental conditions and behavioral discrimination thresholds measured in Expts. 1a and 2a.

Mean response strength of the right temporal cluster (i.e., the mean scalar value, or current density, of the solution points showing the significant difference between the conditions) averaged across the two perceived pitch conditions positively correlated with the participant's sensitivity in pitch discrimination measured before the experiment (two-tailed Pearson's $r: r_{(14)}=$ $0.59 ; p=0.02$ ). There was no significant correlation between the activity of the right frontal network and behavioral discrimination proficiency $(p=0.4)$. No significant correlations were found between brain activity and behavior in Expt. $2(p=0.4)$. These negative results of the correlational analyses for Expt. 2 could follow from a lack of statistical power due to the limited number of participants in Expt. 2 as compared to Expt. 1.

\section{Discussion}

The single-trial EEG topographic analysis predicted the subjective perception of identical tones with above-chance accuracy for the majority of the participants. This suggests that random variations in the configuration of the brain networks responding to basic sounds determine the resulting pitch percepts, independently of the physical stimulation.

Moreover, we identified when and where in the brain the occurrence of fluctuations in activity modulated perceived pitch by contrasting electrical neuroimaging analyses of AEPs to the sounds that were judged higher in pitch versus AEPs to identical sounds that were judged lower in pitch. AEPs modulated topographically over the 93-109 ms poststimulus interval, indicative of the engagement of distinct configurations of active brain networks.

Electrical neuroimaging analyses contrasting responses to identical tones as a function of perceived pitch indicated the locations and the periods critical for subjective pitch perception. 
a ERPs Waveform and Time-Wise Statistical Differences

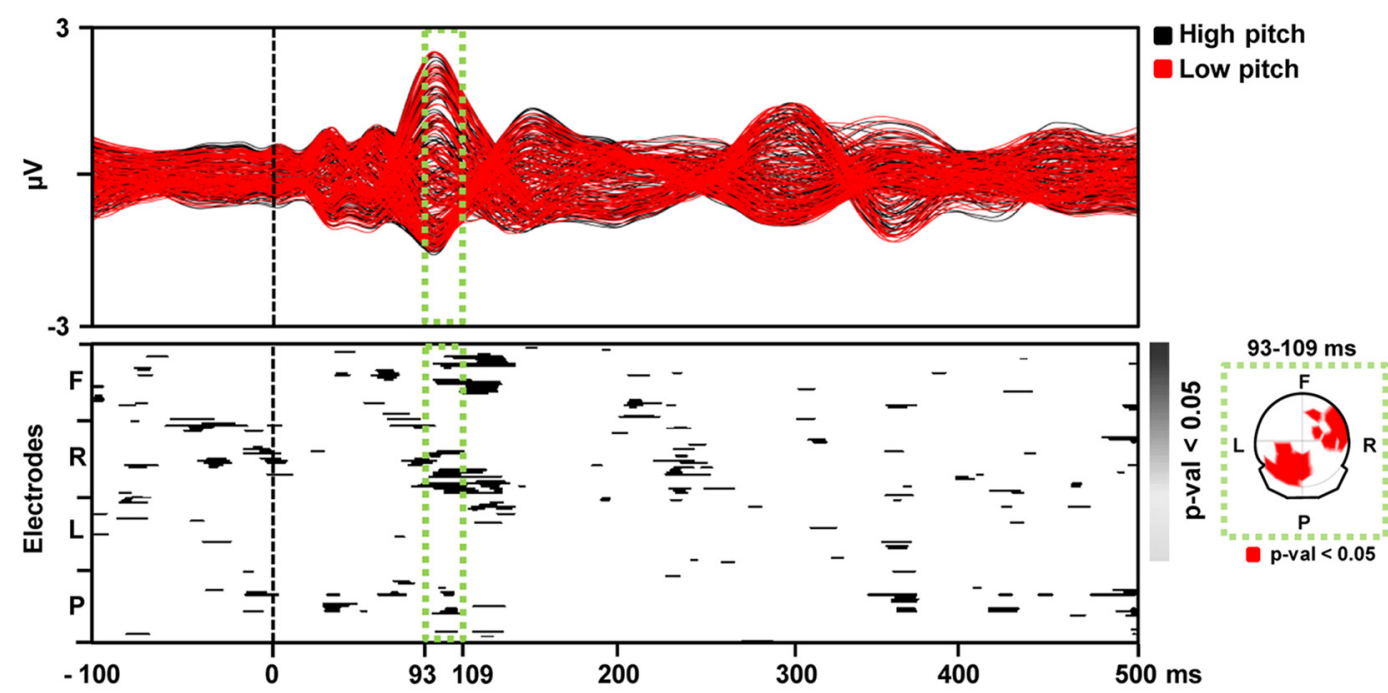

b Time-Wise Global Field Power Statistical Difference

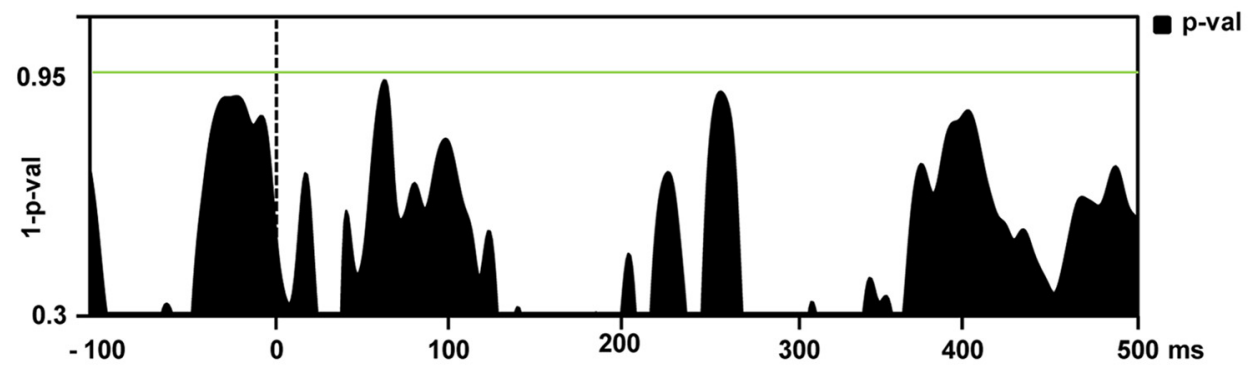

c Time-Wise Topographic Statistical Difference

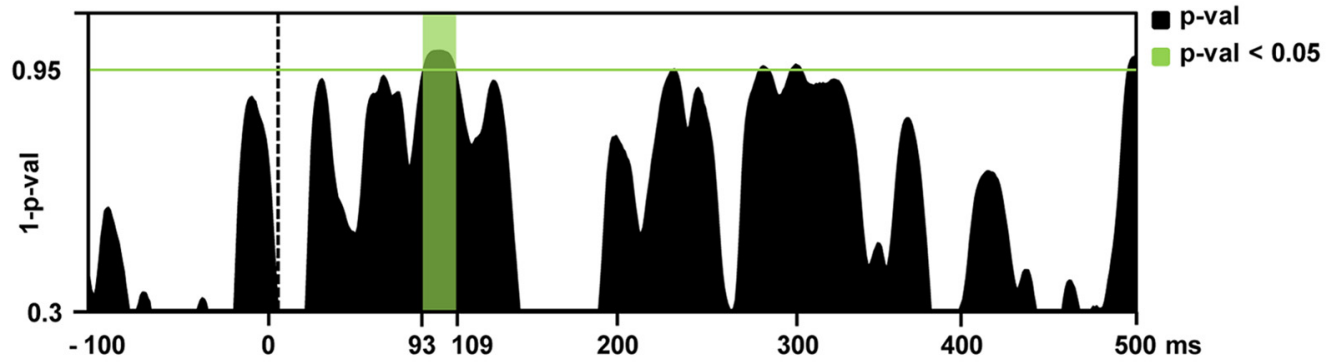

d LAURA Source Estimation Statistical Difference (93-109ms)

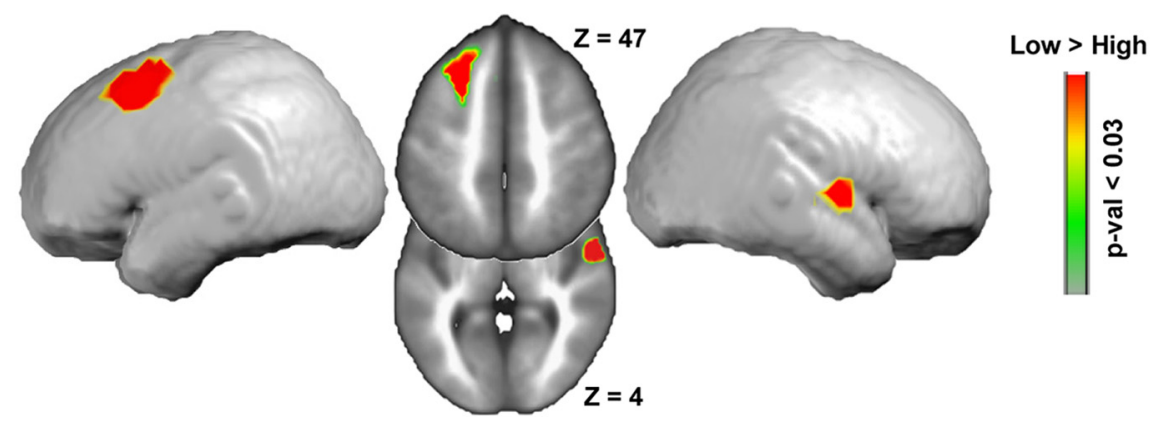

Figure 3. $\boldsymbol{a}$, Top, The ERP to the low (red) and high (black) perceived pitch conditions are displayed in microvolts as a function of time relative to sound onset. The time period showing significant $(p<0.05)$ topographic difference between the conditions is indicated in green. Bottom, The results of the timewise paired $t$ tests at each of the scalp electrodes from the group-averaged ERP waveforms are shown (only $p<0.05$ with an 11 time-frame temporal criterion are shown). In the green box, the scalp topography of the significant differences for the 93-109 ms period after stimulus onset represented nasion upward. $\boldsymbol{b}$, Results of the timewise paired $t$ tests on the global field power contrasting "high" versus "low" perceived pitch conditions. Period of significant $(p<0.05 ; 11$ time-frame temporal criterion) differences in response strength are marked in green. $c$, Results of the timewise analysis of the global dissimilarity contrasting "high" versus "low" perceived pitch conditions. Period of significant $(p<0.05 ; 11$ time-frame temporal criterion) topographic differences are marked in green. $\boldsymbol{d}$, Distributed LAURA source estimations. Statistical contrast (paired ttest) across all solution points during the period of topographic modulation (93-109 ms after stimulus onset) reveals a significantly stronger ( $p<0.03)$ activation of the left middle frontal gyrus and right superior temporal gyrus in the "low" versus "high" perceived pitch condition. 
a ERPs Waveform and Time-Wise Statistical Differences

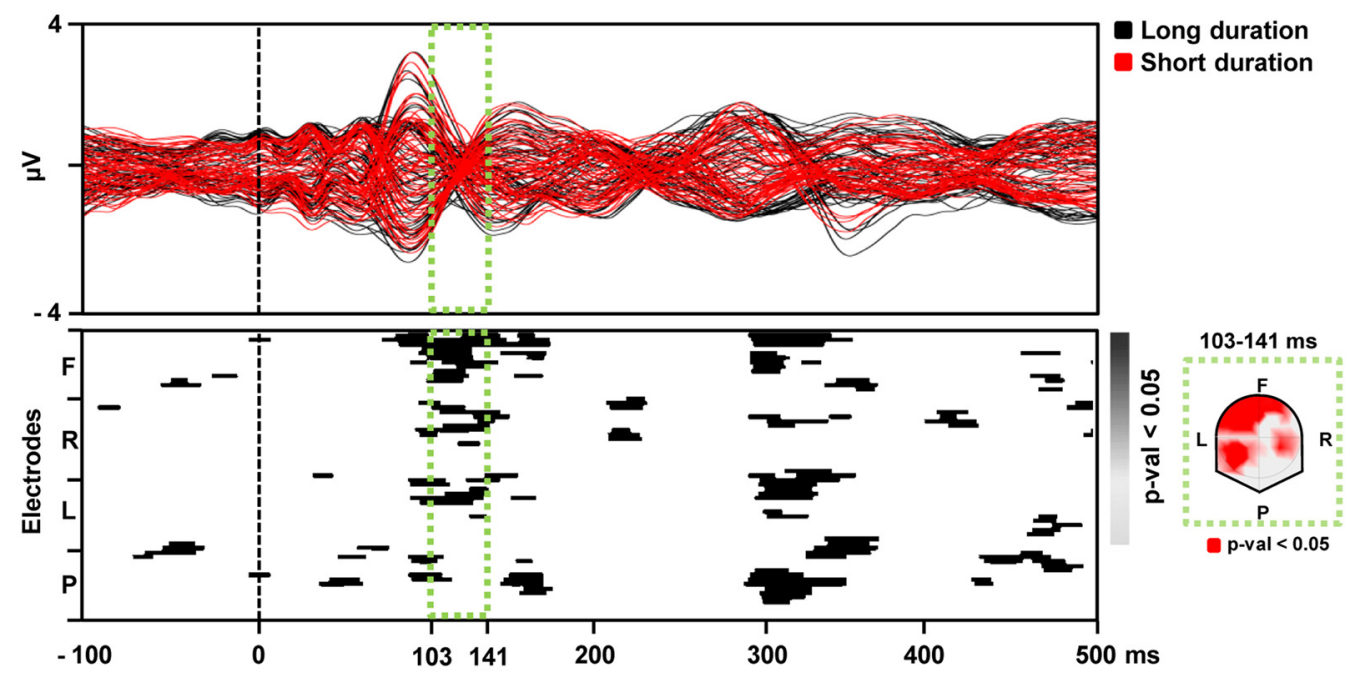

b Time-Wise Global Field Power Statistical Difference

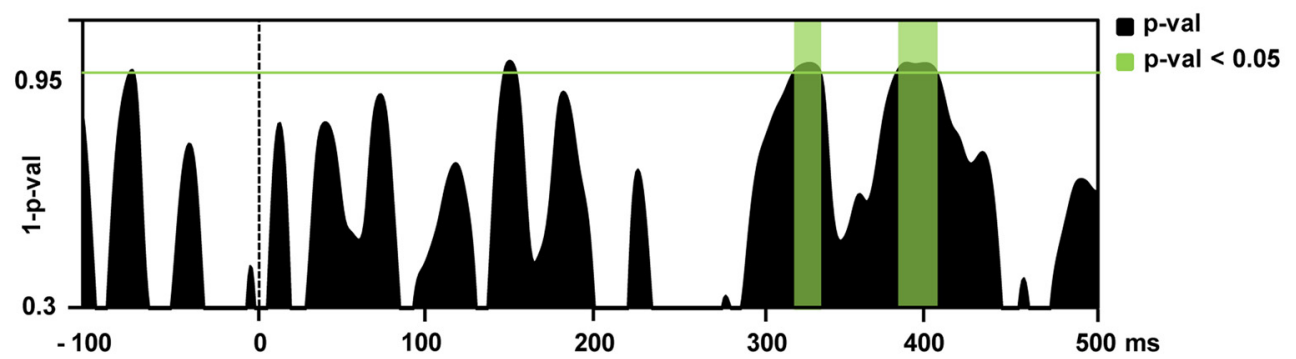

C Time-Wise Topographic Statistical Difference

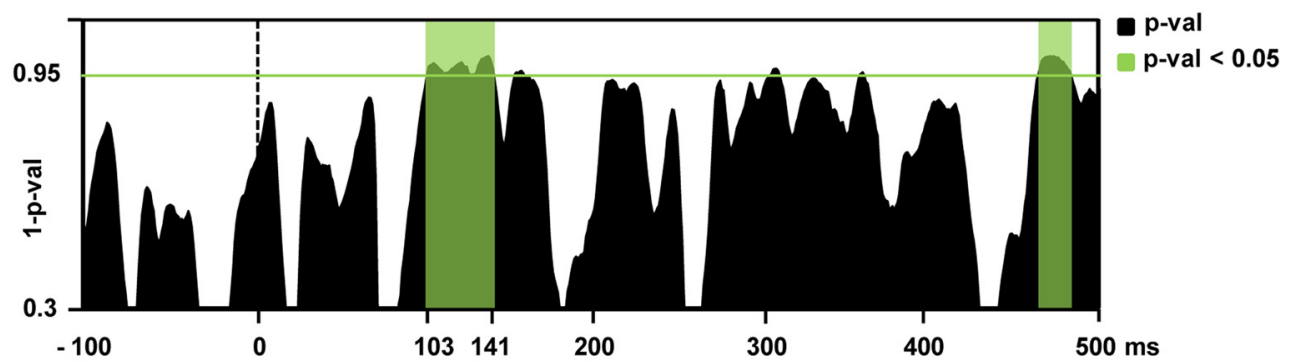

d LAURA Source Estimation Statistical Difference (103-141ms)
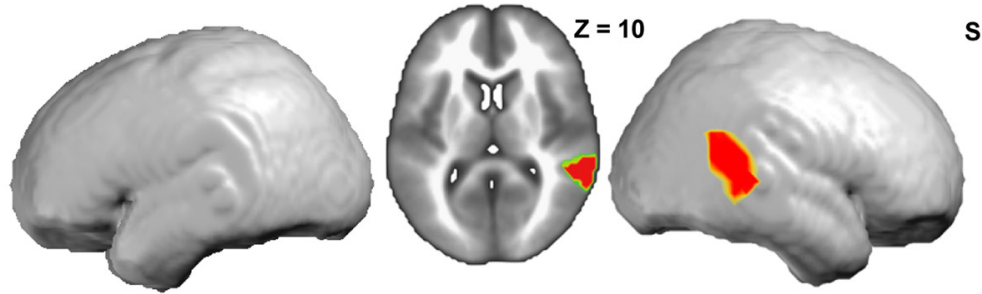

Short > Long

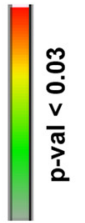

Figure 4. $\quad \boldsymbol{a}$, Top, The ERP to the short (red) and long (black) perceived duration conditions are displayed in microvolts as a function of time relative to sound onset. The time period showing significant $(p<0.05)$ topographic difference between the conditions is indicated in green. Bottom, The results of the timewise paired $t$ tests at each of the scalp electrodes from the group-averaged ERP waveforms are shown (only $p<0.05$ with a 11 time-frame temporal criterion are shown). In the green box, the scalp topography of the significant differences for the 103-141 ms period after stimulus onset, represented nasion upward. $\boldsymbol{b}$, Results of the timewise paired $t$ tests on the global field power contrasting "short" versus "long" perceived duration conditions. Period of significant $(p<0.05 ; 11$ time-frame temporal criterion) differences in response strength are marked in green. $c$, Results of the timewise analysis of the global dissimilarity contrasting "short" versus "long" perceived duration conditions. Period of significant $(p<0.05 ; 11$ time-frame temporal criterion) topographic differences are marked in green. $\boldsymbol{d}$, Distributed LAURA source estimations. Statistical contrast (paired $t$ test) across all solution points during the period of topographic modulation (103-141 ms after stimulus onset) reveals a significantly stronger ( $p<0.03)$ activation of the right temporoparietal junction in the "short" versus "long" perceived duration condition. 
Thus, using the same approach but asking participants to discriminate the identical sounds according to another auditory dimension should reveal the critical spatiotemporal pattern of activity involved in experiencing this other dimension. To this end, we conducted a second EEG experiment involving the same stimuli as in Experiment 1b, but with different instructions. Participants now indicated which of the two identical stimuli was of longer duration instead of higher pitch. The single-trial EEG topographic classification algorithm reliably predicted perceptual outcome. AEPs modulated topographically as a function of the perceived duration of the identical tones over the 103-141 ms poststimulus interval. Distributed linear source estimations calculated over this period revealed significantly stronger activation within the right temporoparietal junction for stimuli perceived as longer duration versus shorter duration.

There was no evidence for prestimulus differences between the AEP to the two perceived pitch or perceived duration conditions, in terms of either global response strength or topography. This result indicates that differential perceptions of the pitch or duration of identical stimuli are unlikely the consequence of variation in prestimulus brain state (no prestimulus baseline correction was applied to the AEPs) as could have been hypothesized based on reports for interaction between stimulus-related evoked activity and the preceding ongoing brain activity (Arieli et al., 1996; Sadaghiani et al., 2009). We cannot rule out, however, that non-phase-locked prestimulus oscillatory activity differed between conditions as in, e.g., Romei et al. (2008), because our analyses were time-locked to the onset of the stimuli. Rather, our results suggest that the differences between the contrasted conditions were linked to random fluctuations of activity occurring at specific stages of the brain responses evoked by the stimuli.

Likewise, because effects of stimulus order within each pair were controlled, mere habituation or priming effects unlikely account for our results. Furthermore, GFP analyses provided no evidence for poststimulus changes in response strength. Based on evidence that variations in the amount of attention allocated to a stimulus manifest as changes in response gain (i.e., in GFP) without changes in the configuration of the underlying neural generators (i.e., in topography; Hillyard and Anllo-Vento, 1998; Luck et al., 2000), the absence of GFP modulations suggests that our effects were unlikely the consequence of quantitative variations in attention.

The present results are distinct from the literature reporting differential behavioral and brain responses to physically identical stimuli, including phenomena associated with binocular rivalry (Tong et al., 2006), ambiguous multistable stimuli (Haynes et al., 2005; Sterzer et al., 2009; Britz et al., 2011), or variations in the instruction about which stimuli characteristics participants had to attend (Rinne et al., 2009). The stimuli in all of these cases indeed contained the physical information that participants perceived across the experimental conditions. Dissociations between observers' responses and physical stimuli resulted from spontaneous or experimentally induced modulation of selective attention, in turn determining which stimulus characteristic observers attended to and consciously perceived. For instance, in a recent neuroimaging study on pitch processing, Rinne et al. (2009) showed that task instructions modulated brain responses within low-level auditory cortices to the same stimuli in a condition involving attending to different characteristics of the stimuli, namely pitch differences, or to the sequence between the stimuli. By contrast, the discrimination on the identical stimuli in our study was to be done on the same dimension across the experimental conditions, and the value of this dimension did not change; there was no acoustic information allowing participants to perceive a given stimulus as being of higher or lower pitch or longer or shorter duration. Instead, participants could only perceive differences along pitch or duration dimensions based on the "erroneous" activation of neuronal populations, which we hypothesize is due to random fluctuations in brain activity (i.e., brain noise), though we cannot unequivocally exclude some contribution from sources of external noise.

Brain noise occurs at various stages along the sensory processing pathway, including the transduction of physical to nervous signal (Lillywhite and Laughlin, 1979) or neural response (de Ruyter van Steveninck et al., 1997; for review, see Faisal et al., 2008). As demonstrated by the significantly above-chance level of the accuracy of the single-trial classification analyses, random variations in brain responses to the sounds were principal contributors to the changes in perceptual outcome. These fluctuations in brain responses to the identical tones involved sufficiently large neural ensembles to be measured at the level of the global topography of the electric field recorded at the surface of the scalp.

The early timing of our effect and the finding that differences between the conditions manifested within low-level brain areas suggest that our effects relate to representation of the stimuli pitch and duration dimensions rather than to comparison mechanisms signaling differences between pitch and duration representations. In the pitch experiment, the statistical contrast of the source estimations of AEP to higher versus lower perceived pitch condition identified modulations within a right anterior temporal and left middle frontal networks at $\sim 100 \mathrm{~ms}$ after stimulus onset. With regard to current models of the spatial and temporal hierarchy of pitch processing, the present effects occur within pitch-sensitive areas at timing linked to the processing of pitch (e.g., Zatorre et al., 1992, 1994; Griffiths et al., 1998, 2010; Griffiths, 2001; Lütkenhöner et al., 2001; Patterson et al., 2002; Seither-Preisler et al., 2006; Hyde et al., 2008; Schönwiesner and Zatorre, 2008). In addition, our results for the involvement of low-level auditory cortices, at early latencies, in the subjective or perceptual interpretation of stimuli beyond their acoustic information support and extend recent evidence for a role of these structures in interpreting ambiguous phonemic information (Kilian-Hütten et al., 2011).

Random variations in brain responses to the sounds can occur in principle at any stage of sound processing. However, as revealed by contrasting AEPs to sounds perceived as being of higher versus lower pitch, only variations within right temporal and left frontal cortices at $100 \mathrm{~ms}$ after onset were relevant for pitch perception. Likewise, contrasting the responses to the same sounds as a function of variations in the perception of stimulus duration allowed us to reveal the neural underpinnings of subjective duration. While the recent meta-analyses by Wiener et al. (2010) suggest a prominent involvement of the right inferior frontal gyrus and bilateral supplementary motor area in duration representation, our result for a role of the right TPJ in the perception of duration has already been reported (Rao et al., 2001; Schubotz and von Cramon, 2001; Pouthas et al., 2005; Bueti et al., 2008).

The significant positive correlation between the magnitude of the mean activity of the brain region showing a differential response as a function of perceived pitch and discrimination sensitivity corroborate computational models linking perceptual discrimination proficiency with the magnitude of trial-to-trial variability in brain responses to identical stimuli (Neri, 2010), notably including signal detection theory (Macmillan and Creelman, 2005).

Finally, our results provide a potential explanation for the observation that perceptual learning can occur when trained with 
identical stimuli. After observing pitch-discrimination improvement by training with identical stimuli, Amitay et al. (2006) concluded that perceptual learning does not rely on fine-tuning of comparison mechanisms as previously thought because identical stimuli cannot be compared. The authors therefore concluded that performance improvement was mediated by a refinement of stimulus representation or in facilitated access to stimulus representations. Our results that identical stimuli are actually perceived as different and represented by distinct configurations of brain generators challenge this conclusion by indicating that a comparison mechanism could also act on identical stimuli and that its improvement could therefore support perceptual learning (Micheyl et al., 2009).

In line with previous evidence for the dissociations between sensation and perception (in hearing: Hillyard et al., 1971; Parasuraman and Beatty, 1980; in vision: Ress et al., 2000; Ress and Heeger, 2003), we provide neurophysiological evidence for an effect of brain noise at the level of large neural ensembles and its direct influence on perception and sensitivity, the main premises of signal detection theory. Moreover, we show that the neural basis of phenomenological experience can be neuroscientifically investigated without confounds induced by variation in task or physical input, even in very basic, nonambiguous stimuli. This opens an avenue for research on the neural underpinnings of conscious perception along stimulus dimension, independently of physical inputs.

\section{References}

Amitay S, Irwin A, Moore DR (2006) Discrimination learning induced by training with identical stimuli. Nat Neurosci 9:1446-1448.

Arieli A, Sterkin A, Grinvald A, Aertsen A (1996) Dynamics of ongoing activity: explanation of the large variability in evoked cortical responses. Science 273:1868-1871.

Ary JP, Klein SA, Fender DH (1981) Location of sources of evoked scalp potentials: corrections for skull and scalp thicknesses. IEEE Trans Biomed Eng 28:447-452.

Britz J, Landis T, Michel CM (2009) Right parietal brain activity precedes perceptual alternation of bistable stimuli. Cereb Cortex 19:55-65.

Britz J, Pitts MA, Michel CM (2011) Right parietal brain activity precedes perceptual alternation during binocular rivalry. Hum Brain Mapp 32:1432-1442.

Brunet D, Murray MM, Michel CM (2011) Spatiotemporal analysis of multichannel EEG: CARTOOL. Comput Intell Neurosci 2011:813870.

Bueti D, van Dongen EV, Walsh V (2008) The role of superior temporal cortex in auditory timing. PLoS One 3:e2481.

De Lucia M, Michel CM, Clarke S, Murray MM (2007) Single-trial topographic analysis of human EEG: a new 'image' of event-related potentials. Proceedings Information Technology Applications in Biomedicine.

De Lucia M, Michel CM, Murray MM (2010) Comparing ICA-based and single-trial topographic ERP analyses. Brain Topogr 23:119-127.

de Ruyter van Steveninck RR, Lewen GD, Strong SP, Koberle R, Bialek W (1997) Reproducibility and variability in neural spike trains. Science 275:1805-1808.

Faisal AA, Selen LP, Wolpert DM (2008) Noise in the nervous system. Nat Rev Neurosci 9:292-303.

Grave de Peralta Menendez R, Gonzalez Andino S, Lantz G, Michel CM, Landis T (2001) Noninvasive localization of electromagnetic epileptic activity. I. Method descriptions and simulations. Brain Topogr 14:131-137.

Grave de Peralta Menendez R, Murray MM, Michel CM, Martuzzi R, Gonzalez Andino SL (2004) Electrical neuroimaging based on biophysical constraints. Neuroimage 21:527-539.

Green DM, Swets JA (1966) Signal detection theory and psychophysics. New York: Wiley.

Griffiths TD (2001) The neural processing of complex sounds. Ann N Y Acad Sci 930:133-142.

Griffiths TD, Büchel C, Frackowiak RS, Patterson RD (1998) Analysis of temporal structure in sound by the human brain. Nat Neurosci $1: 422-427$.
Griffiths TD, Kumar S, Sedley W, Nourski KV, Kawasaki H, Oya H, Patterson RD, Brugge JF, Howard MA (2010) Direct recordings of pitch responses from human auditory cortex. Curr Biol 20:1128-1132.

Guthrie D, Buchwald JS (1991) Significance testing of difference potentials. Psychophysiology 28:240-244.

Haynes JD, Deichmann R, Rees G (2005) Eye-specific effects of binocular rivalry in the human lateral geniculate nucleus. Nature 438:496-499.

Hillyard SA, Anllo-Vento L (1998) Event-related brain potentials in the study of visual selective attention. Proc Natl Acad Sci U S A 95:781-787.

Hillyard SA, Squires KC, Bauer JW, Lindsay PH (1971) Evoked potential correlates of auditory signal detection. Science 172:1357-1360.

Hyde KL, Peretz I, Zatorre RJ (2008) Evidence for the role of the right auditory cortex in fine pitch resolution. Neuropsychologia 46:632-639.

Kilian-Hütten N, Vroomen J, Formisano E (2011) Brain activation during audiovisual exposure anticipates future perception of ambiguous speech. Neuroimage 57:1601-1607.

Koenig T, Melie-García L (2010) A method to determine the presence of averaged event-related fields using randomization tests. Brain Topogr 23:233-242.

Lehmann D (1987) Principles of spatial analysis. In: Handbook of electroencephalography and clinical neurophysiology, Vol 1, Methods of analysis of brain electrical and magnetic signals (Gevins AS, Remond A, eds), pp 309-354. Amsterdam: Elsevier.

Lehmann D, Skrandies W (1980) Reference-free identification of components of checkerboard-evoked multichannel potential fields. Electroencephalogr Clin Neurophysiol 48:609-621.

Lillywhite PG, Laughlin SB (1979) Transducer noise in a photoreceptor. Nature 277:569-572.

Luck SJ, Woodman GF, Vogel EK (2000) Event-related potential studies of attention. Trends Cogn Sci 4:432-440.

Lütkenhöner B, Lammertmann C, Knecht S (2001) Latency of auditory evoked field deflection N100m ruled by pitch or spectrum? Audiol Neurootol 6:263-278.

Macmillan NA, Creelman CD (2005) Detection theory: a user's guide, Ed 2. New York: Erlbaum.

Michel CM, Murray MM, Lantz G, Gonzalez S, Spinelli L, Grave de Peralta R (2004) EEG source imaging. Clin Neurophysiol 115:2195-2222.

Micheyl C, McDermott JH, Oxenham AJ (2009) Sensory noise explains auditory frequency discrimination learning induced by training with identical stimuli. Atten Percept Psychophys 71:5-7.

Murray MM, Brunet D, Michel CM (2008) Topographic ERP analyses: a step-by-step tutorial review. Brain Topogr 20:249-264.

Murray MM, De Lucia M, Brunet D, Michel CM (2009) Principles of topographic analyses of electrical neuroimaging. In: Event-related potentials II: advances in ERP, EEG, and MEG analysis (Handy TC, ed). Cambridge, MA: MIT Press.

Neri P (2010) How inherently noisy is human sensory processing? Psychon Bull Rev 17:802-808.

Oldfield RC (1971) The assessment and analysis of handedness: the Edinburgh inventory. Neuropsychologia 9:97-113.

Parasuraman R, Beatty J (1980) Brain events underlying detection and recognition of weak sensory signals. Science 210:80-83.

Patterson RD, Uppenkamp S, Johnsrude IS, Griffiths TD (2002) The processing of temporal pitch and melody information in auditory cortex. Neuron 36:767-776.

Perrin F, Pernier J, Bertrand O, Giard MH, Echallier JF (1987) Mapping of scalp potentials by surface spline interpolation. Electroencephalogr Clin Neurophysiol 66:75-81.

Pouthas V, George N, Poline JB, Pfeuty M, Vandemoorteele PF, Hugueville L, Ferrandez AM, Lehéricy S, Lebihan D, Renault B (2005) Neural network involved in time perception: an fMRI study comparing long and short interval estimation. Hum Brain Mapp 25:433-441.

Rao SM, Mayer AR, Harrington DL (2001) The evolution of brain activation during temporal processing. Nat Neurosci 4:317-323.

Ress D, Heeger DJ (2003) Neuronal correlates of perception in early visual cortex. Nat Neurosci 6:414-420.

Ress D, Backus BT, Heeger DJ (2000) Activity in primary visual cortex predicts performance in a visual detection task. Nat Neurosci 3:940-945.

Rinne T, Koistinen S, Salonen O, Alho K (2009) Task-dependent activations of human auditory cortex during pitch discrimination and pitch memory tasks. J Neurosci 29:13338-13343.

Romei V, Brodbeck V, Michel C, Amedi A, Pascual-Leone A, Thut G (2008) 
Spontaneous fluctuations in posterior alpha-band EEG activity reflect variability in excitability of human visual areas. Cereb Cortex 18:2010-2018.

Sadaghiani S, Hesselmann G, Kleinschmidt A (2009) Distributed and antagonistic contributions of ongoing activity fluctuations to auditory stimulus detection. J Neurosci 29:13410-13417.

Schönwiesner M, Zatorre RJ (2008) Depth electrode recordings show double dissociation between pitch processing in lateral Heschl's gyrus and sound onset processing in medial Heschl's gyrus. Exp Brain Res 187:97-105.

Schubotz RI, von Cramon DY (2001) Interval and ordinal properties of sequences are associated with distinct premotor areas. Cereb Cortex 11:210-222.

Seither-Preisler A, Patterson RD, Krumbholz K, Seither S, Lütkenhöner B (2006) From noise to pitch: transient and sustained responses of the auditory evoked field. Hear Res 218:50-63.

Spinelli L, Andino SG, Lantz G, Seeck M, Michel CM (2000) Electromagnetic inverse solutions in anatomically constrained spherical head models. Brain Topogr 13:115-125.

Sterzer P, Jalkanen L, Rees G (2009) Electromagnetic responses to invisible face stimuli during binocular suppression. Neuroimage 46:803-808.
Talairach J, Tournoux P (1988) Co-planar stereotaxic atlas of the human brain. New York: Thieme.

Tong F, Meng M, Blake R (2006) Neural bases of binocular rivalry. Trends Cogn Sci 10:502-511.

Tzovara A, Murray MM, Plomp G, Herzog MH, Michel CM, De Lucia M (2011a) Decoding stimulus-related information from single-trial EEG responses based on voltage topographies. Pattern Recognit. Advance online publication. doi:10.1016/j.patcog.2011.04.007.

Tzovara A, Murray MM, Michel CM, De Lucia M (2011b) A tutorial review of electrical neuroimaging from group-average to single-trial eventrelated potentials. Dev Neuropsychol in press.

van Dijk P, Backes WH (2003) Brain activity during auditory backward and simultaneous masking tasks. Hear Res 181:8-14.

Wiener M, Turkeltaub P, Coslett HB (2010) The image of time: a voxel-wise meta-analysis. Neuroimage 49:1728-1740.

Zatorre RJ, Evans AC, Meyer E, Gjedde A (1992) Lateralization of phonetic and pitch discrimination in speech processing. Science 256:846-849.

Zatorre RJ, Evans AC, Meyer E (1994) Neural mechanisms underlying melodic perception and memory for pitch. J Neurosci 14:1908-1919. 